\title{
HIGH-ORDER EXPONENTIALLY FITTED DIFFERENCE SCHEMES FOR SINGULARLY PERTURBED TWO-POINT BOUNDARY VALUE PROBLEMS*
}

\author{
MILJENKO MARUŠIĆ ${ }^{\dagger}$
}

\begin{abstract}
We introduce a family of exponentially fitted difference schemes of arbitrary order as numerical approximations to the solution of a singularly perturbed two-point boundary value problem: $\varepsilon y^{\prime \prime}+b y^{\prime}+c y=f$. The difference schemes are derived from interpolation formulae for exponential sums. The so-defined $k$-point differentiation formulae are exact for functions that are a linear combination of $1, x, \ldots, x^{k-2}, \exp (-\rho x)$. The parameter $\rho$ is chosen from the asymptotic behavior of the solution in the boundary layer. This approach allows a construction of the method with arbitrary order of consistency. Using an estimate for the interpolation error, we prove consistency of all the schemes from the family. The truncation error is bounded by $C h^{k-2}$, where $C$ is a constant independent of $\varepsilon$ and $h$. Therefore, the order of consistency for the $k$-point scheme is $k-2(k \geq 3)$ in case of a small perturbation parameter $\varepsilon$. There is no general proof of stability for the proposed schemes. Each scheme has to be considered separately. In the paper, stability, and therefore convergence, is proved for three-point schemes in the case when $c<0$ and $b \neq 0$.
\end{abstract}

Key words. difference scheme, singular perturbation, ODE, interpolation, exponential sum

AMS subject classifications. 65L12, 65L11, 65L10, 65L20, 41A30, 65D25

1. Introduction. In this paper we study the numerical solution of the singularly perturbed two-point boundary value problem for the ODE:

$$
\begin{aligned}
(\mathcal{L} u)(x):= & \varepsilon u^{\prime \prime}(x)+b(x) u^{\prime}(x)+c(x) u(x)=f(x), \\
& u(0)=\alpha_{0}, \quad u(1)=\alpha_{1} .
\end{aligned}
$$

We assume that the perturbation parameter $\varepsilon$ and the coefficient $c$ satisfy

$$
\varepsilon>0 \quad \text { and } \quad c(x) \leq 0 \quad \text { for } x \in[0,1] .
$$

It is known that classical methods fail for this problem when $\varepsilon$ is small relative to the mesh width. Various numerical approaches have been proposed, for example, difference schemes $[5,13,15]$ and collocation methods $[2,6,8,9,12]$. An extensive review of methods is given by Kadalbayo and Patidar in [7].

To derive a difference scheme for the above problem, we consider functions that satisfy a differential equation of the form

$$
\left(D-\rho_{1}\right)\left(D-\rho_{2}\right) \cdots\left(D-\rho_{k}\right) s=0
$$

for some given real numbers $\rho_{1}, \rho_{2}, \ldots, \rho_{k}$. Here, $D$ stands for the differentiation operator ( $D f=f^{\prime}=d f / d x$ for some function $f$ ). For mutually distinct $\rho_{i}$, the solution of the above equation is an exponential sum

$$
\sum_{i=1}^{k} \gamma_{i} \mathrm{e}^{\rho_{i} x} .
$$

Generally, if some $\rho_{i}$ are equal, we do not only have exponentials $\exp \left(\rho_{i} x\right)$ in the sum but also functions of the form $x \exp \left(\rho_{i} x\right), x^{2} \exp \left(\rho_{i} x\right), \ldots$ The resulting expressions are

* Received July 25, 2016. Accepted June 4, 2018. Published online on September 11, 2018. Recommended by M. Gander. Work supported by the grant \#037-0982913-2762 of the Croatian Ministry of Science, Education and Sports.

${ }^{\dagger}$ Department of Mathematics, University of Zagreb, Bijenička c. 30, 10000 Zagreb, Croatia (miljenko.marusic@math.hr). 
often called extended exponential sums [3] to be distinguished from the proper exponential sums (1.4). In this paper we consider extended exponential sums, and we will simply refer to them as exponential sums. The so-defined method belongs to a class of exponentially fitted methods. Exponential fitting is a well-known approach widely used for singularly perturbed problems [7].

The novelty of this paper is a new approach for the derivation of difference schemes. Using interpolation formulae, we approximate the derivatives in the differential equation (1.1) and obtain a difference scheme. The consistency of the schemes follows directly from the estimate for the interpolation error of the exponential sum. The presented results give an algorithm for the derivation of consistent schemes of arbitrary order. Unfortunately, we do not have a general result of stability (and convergence) for the proposed schemes. So, in this paper, we consider stability of three-point schemes and prove that they are first-order convergent in the singular perturbation case.

In the forthcoming sections we present basic facts about exponential sums, explain the derivation of difference schemes, prove consistency of the schemes, and analyze the convergence for three-point schemes. Finally, we exemplify our methods at several singularly perturbed problems.

2. Exponentially fitted differentiation formulae. As an approximation for $u(x)$, we consider interpolation by particular classes of exponential sums. Since a solution of (1.1)-(1.2) asymptotically behaves as an exponential function on the boundary layer, we just use one exponential term. Hence, we use an exponential sum from the null-space of the differential operator

$$
D^{k-1}(D+\rho) s=0
$$

and our exponential sum is of the form

$$
s(x)=\sum_{i=0}^{k-2} \gamma_{i} x^{i}+\gamma_{k-1} \mathrm{e}^{-\rho x} .
$$

Let be given a real number $\rho$, an increasing sequence of points $\left(t_{i}\right)_{0}^{k-1}$ $\left(t_{0}<t_{1}<\ldots<t_{k-1}\right)$, and a sequence of real numbers $\left(y_{i}\right)_{0}^{k-1}$. Then, there is a unique exponential sum (2.2) satisfying the interpolation conditions

$$
s\left(t_{i}\right)=y_{i}, \quad i=0, \ldots, k-1 .
$$

Interpolation by exponential sums is widely investigated in [3] and the references therein.

It is more convenient to write an exponential sum in Lagrange form. Let $L_{j}^{k, m}$ be an exponential sum from the null-space of the differential operator (2.1) satisfying

$$
L_{j}^{k, m}(i)= \begin{cases}0 & \text { for } i+m \neq j \\ 1 & \text { for } i+m=j\end{cases}
$$

for $j=0, \ldots, k-1, i=-m, \ldots, k-1-m$, and for some $m(0 \leq m \leq k-1)$. For equidistant points $\left(t_{i}\right)_{0}^{k-1}\left(t_{i+1}=t_{i}+h, h>0\right)$, the exponential sum

$$
s(x)=\sum_{j=0}^{k-1} L_{j}^{k, m}\left(\frac{x-t_{m}}{h}\right) y_{j},
$$


satisfies the interpolation conditions (2.3). Note that the so-defined $s$ is not from the null-space of (2.1) but from the null-space of

$$
D^{k-1}(D+\rho / h) s=0 .
$$

The interpolation error for exponential sums is studied in [10] and [11]. Here we reveal results for the error bound applied to the sum (2.5).

LemMA 2.1 ([10]). Let $u \in C^{k}(a, b)$, and let $s$ be an exponential sum (2.5) of order $k$ that interpolates $u$ at an equidistant sequence of points $\left(t_{i}\right)_{0}^{k-1},\left(a \leq t_{0}<\ldots<t_{k-1} \leq b\right.$, $\left.h=t_{i+1}-t_{i}\right)$. Then for all $x \in[a, b]$ and for $r=0,1, \ldots, k-1$, the following estimates hold:

$$
\left|u^{(r)}(x)-s^{(r)}(x)\right| \leq h^{k-r} C_{k, r}\left(\rho,\left(x-t_{0}\right) / h\right)\left\|u^{(k)}+\frac{\rho}{h} u^{(k-1)}\right\|_{\infty},
$$

and

$$
\left|u^{(r)}(x)-s^{(r)}(x)\right| \leq h^{k-r-1}|\rho| C_{k, r}\left(\rho,\left(x-t_{0}\right) / h\right)\left\|\frac{h}{\rho} u^{(k)}+u^{(k-1)}\right\|_{\infty},
$$

for $r=0,1, \ldots, k-1$. The function $C_{k, r}(\rho, y)$ satisfies

$$
\begin{array}{lll}
0 & <C_{k, r}(0, y)<\infty, & \\
0<\lim _{\rho \rightarrow \infty} \rho C_{k, r}(\rho, y)<\infty, & r=0,1, \ldots, k-1, \quad 0<y, \\
0<\lim _{\rho \rightarrow \infty} \rho^{1-r} C_{k, r}(\rho, y)<\infty, & r=0,1, \ldots, k-1, \quad y=0, \\
0<\lim _{\rho \rightarrow-\infty}|\rho| C_{k, r}(\rho, y)<\infty, & r=0,1, \ldots, k-1, \quad y<k-1, \\
0<\lim _{\rho \rightarrow-\infty}|\rho|^{1-r} C_{k, r}(\rho, y)<\infty, & r=0,1, \ldots, k-1, \quad y=k-1 .
\end{array}
$$

Here, by \|\|$_{\infty}$ we denoted the maximum norm on the segment $[a, b]$.

For $\rho=0$, the exponential sum is a polynomial of order $k$. In this case, the interpolation error is bounded by $h^{k} C\left\|u^{(k)}\right\|_{\infty}$. When $\rho \rightarrow \infty$, the exponential sum tends to a polynomial of order $k-1$, and the bound reads $h^{k-1} C\left\|u^{(k-1)}\right\|_{\infty}$. This is in concordance with results for the interpolation by polynomial functions.

However, when $u$ is a solution of the singularly perturbed problem (1.1)-(1.2), due to the exponential behavior at the boundary layer, $\left\|u^{(k)}\right\|_{\infty}$ is not bounded for small $\varepsilon$. For $b(x)<0$, the asymptotic behavior is described by (cf. [15])

$$
\left|u^{(l)}(x)\right| \leq C\left[1+\varepsilon^{-l} \exp \left(-b_{\min } \frac{1-x}{\varepsilon}\right)\right],
$$

where

$$
b_{\min }=\min _{x \in[0,1]}|b(x)|
$$

and $C$ is a constant independent of $\varepsilon$ and $x$. When $b(x)>0$, an analogous result holds (just substitute $x \mapsto 1-x$ ). Further, a solution $u$ may be bounded in the similar manner when $b \equiv 0$.

The interpolation error when $u$ is a solution of a singularly perturbed problem is studied in [11]; the main result is reported in the following lemma. 
LEMMA 2.2 ([11]). Let u be a solution of a singularly perturbed boundary value problem (1.1)-(1.2), and let $\left(x_{i}\right)_{0}^{n}$ be an equidistant sequence of points $\left(0=x_{0}<x_{1}<\ldots<x_{n}=1\right.$, $\left.h=x_{i}-x_{i-1}\right)$ for an arbitrarily chosen integer $n$ satisfying

$$
h \geq 4(k-1) \varepsilon \ln (1 / \varepsilon) / b_{\min },
$$

where the constant $b_{\min }$ is defined by (2.6). Further, assume that

1. The functions $b, c$, and $f$ are sufficiently smooth such that $u \in C^{k}(0,1)$;

2. $b(x) \neq 0$ and $c(x) \leq 0$ for all $x \in[0,1]$;

3. The parameter $\rho$ from the exponential part of the exponential sum is of the same sign as the function $b$ and satisfies $K \leq h /(|\rho| \varepsilon) \leq b_{\min }^{-1}$ for some positive constant $K$.

Then the exponential sum $s$ of order $k(k \geq 2)$ that interpolates the solution $u$ at $k$ consecutive mesh points (a subsequence of $\left(x_{i}\right)_{0}^{n}$ denoted by $\left(t_{i}\right)_{1}^{k}$ ) satisfies

$$
\left|u^{(r)}(x)-s^{(r)}(x)\right| \leq R h^{k-1-r}, \quad r=0,1, \ldots, k-1
$$

for all $x \in\left[t_{1}, t_{k-1}\right]$ when $b(x)<0$ or all $x \in\left[t_{2}, t_{k}\right]$ when $b(x)>0$. The constant $R$ is independent of $h$ and $\varepsilon$. Further,

$$
\lim _{\varepsilon \rightarrow 0}\left|u^{(r)}(x)-s^{(r)}(x)\right| \leq R h^{k-1-r}, \quad r=0,1, \ldots, k-1,
$$

for all $x \in\left[t_{1}, t_{k}\right)$ when $b(x)<0$ or all $x \in\left(t_{1}, t_{k}\right]$ when $b(x)>0$.

Note that Lemma 2.2 assumes $b(x) \neq 0$. A similar result may be obtained for the self-adjoint problem $(b \equiv 0)$.

3. A derivation of $k$-points difference schemes. To discretize the differential equation (1.1)-(1.2) we divide the interval $[0,1]$ into $n$ equal subintervals

$$
0=x_{0}<x_{1}<\cdots<x_{n-1}<x_{n}=1, \quad h=1 / n, \quad x_{i}=i h .
$$

A finite difference method comprises a discretization of the differential equation using the grid points $x_{i}$, where the unknown function $u$ is approximated by an exponential sum of order $k$ in the neighborhood of the knot $x_{i}$.

For given $k(k \geq 3)$ and arbitrary $i \in\{0, \ldots, n\}$, we choose $m_{i} \in\{0,1, \ldots, k-1\}$ satisfying $0 \leq i-m_{i} \leq n-k+1$. Let $s_{i}^{k, m_{i}}$ denote an exponential sum defined over $k$ consecutive grid points $x_{i-m_{i}}, \ldots, x_{i+k-m_{i}-1}$. By taking advantage of the equidistant mesh, we may apply the representation of exponential sums given by (2.4) and (2.5):

$$
s_{i}^{k, m_{i}}(x)=\sum_{j=-m_{i}}^{k-m_{i}-1} L_{j}^{k, m_{i}}\left(\frac{x-x_{i}}{h}\right) u_{j} .
$$

The unknowns $u_{i}$ are determined by the approximation of $u\left(x_{i}\right), u^{\prime}\left(x_{i}\right)$, and $u^{\prime \prime}\left(x_{i}\right)$ in (1.1) with $s_{i}^{k, m_{i}}\left(x_{i}\right),\left(s_{i}^{k, m_{i}}\right)^{\prime}\left(x_{i}\right)$, and $\left(s_{i}^{k, m_{i}}\right)^{\prime \prime}\left(x_{i}\right)$, respectively:

$$
\left(\mathcal{L} s_{i}^{k, m_{i}}\right)\left(x_{i}\right) \equiv \varepsilon\left(s_{i}^{k, m_{i}}\right)^{\prime \prime}\left(x_{i}\right)+b_{i}\left(s_{i}^{k, m_{i}}\right)^{\prime}\left(x_{i}\right)+c_{i} s_{i}^{k, m_{i}}\left(x_{i}\right)=f_{i} .
$$

Here we used the abbreviations $b_{i}=b\left(x_{i}\right), c_{i}=c\left(x_{i}\right)$, and $f_{i}=f\left(x_{i}\right)$. Now,

$$
\left.\mathcal{L}\left[L_{j}^{k, m_{i}}\left(\frac{x-x_{i}}{h}\right)\right]\right|_{x=x_{i}}=\frac{\varepsilon}{h^{2}}\left(L_{j}^{k, m_{i}}\right)^{\prime \prime}(0)+\frac{b_{i}}{h}\left(L_{j}^{k, m_{i}}\right)^{\prime}(0)+c_{i} \equiv\left(\mathcal{L}_{i} L_{j}^{k, m_{i}}\right)(0)
$$


leads to the equation

$$
\sum_{j=-m_{i}}^{k-m_{i}-1}\left(\mathcal{L}_{i} L_{j}^{k, m_{i}}\right)(0) u_{i+j}=f_{i} .
$$

Since the boundary conditions (1.2) give two equations

$$
u_{0}=\alpha_{0}, \quad u_{n}=\alpha_{1},
$$

we need $n-1$ equations of the form (3.1) to determine the $n+1$ unknowns $u_{j}$.

Not all of the choices for such equations will lead to a regular system of equations or to a stable method. However, all methods are consistent as we prove in the next section. A reasonable strategy for the choice of equations is to select mutually different $x_{i}$ 's. Later in this paper we construct and examine three convergent three-point methods to illustrate our approach.

Up to this point we did not discuss the choice of the parameter $\rho$ from the exponential sum $s_{i}$. We determine $\rho$ from the condition that the scheme is exact when the solution of the problem (1.1)-(1.2) with constant coefficients is an exponential function. In this case, $\rho$ is the largest (by the absolute value) root of the quadratic equation

$$
\frac{\varepsilon}{h^{2}} \rho^{2}-\frac{b_{i}}{h} \rho+c_{i}=0 .
$$

We denote by $\rho_{i}$ the parameter $\rho$ associated with the exponential sum $s_{i}^{k, m_{i}}$.

In the case $b(x) \neq 0, \rho_{i}$ is given by

$$
\rho_{i}= \begin{cases}h \frac{b_{i}+\sqrt{b_{i}^{2}-4 \varepsilon c_{i}}}{2 \varepsilon} & \text { for } b_{i}>0, \\ h \frac{b_{i}-\sqrt{b_{i}^{2}-4 \varepsilon c_{i}}}{2 \varepsilon} & \text { for } b_{i}<0 .\end{cases}
$$

This definition is used for the choice of the tension parameters in the collocation by tension splines [9]. Another possibility is to choose $\rho$ according to an asymptotic expansion of the solution in the boundary layer. This approach is widely used for exponentially fitted difference schemes. Such a choice leads to

$$
\rho_{i}=\frac{h b_{i}}{\varepsilon} .
$$

Both choices (3.4) and (3.5) are hardly distinguishable for small perturbation parameters $\varepsilon$. For $b_{i}>0$ we have

$$
\frac{h}{\varepsilon} \frac{b_{i}+\sqrt{b_{i}^{2}-4 \varepsilon c_{i}}}{2}=\frac{h}{\varepsilon} b_{i}-\frac{2 h c_{i}}{b_{i}+\sqrt{b_{i}^{2}-4 \varepsilon c_{i}}} .
$$

If $c \equiv 0$, the choices (3.4) and (3.5) are equal.

When $b \equiv 0$, the solution of the problem (1.1)-(1.2) has two boundary layers, and $\rho$ is calculated according to

$$
\rho_{i}= \begin{cases}h \sqrt{-\frac{c_{i}}{\varepsilon}} & \text { for } i<\frac{n}{2}, \\ -h \sqrt{-\frac{c_{i}}{\varepsilon}} & \text { for } i \geq \frac{n}{2} .\end{cases}
$$


4. Consistency of the difference schemes. Here we study the application of the exact solution $u$ of the problem (1.1)-(1.2) to the scheme given by (3.1). Let us define

$$
\begin{aligned}
\tau_{i} & =\sum_{j=-m_{i}}^{k-m_{i}-1}\left(\mathcal{L}_{i} L_{j}^{k, m_{i}}\right)(0) u\left(x_{i+j}\right)-\sum_{j=-m_{i}}^{k-m_{i}-1}\left(\mathcal{L}_{i} L_{j}^{k, m_{i}}\right)(0) u_{i+j} \\
& =\sum_{j=-m_{i}}^{k-m_{i}-1}\left(\mathcal{L}_{i} L_{j}^{k, m_{i}}\right)(0) u\left(x_{i+j}\right)-f_{i},
\end{aligned}
$$

where $i$ and $m_{i}$ are arbitrarily chosen.

There is an unique exponential sum $S_{i}^{k, m_{i}}$ of order $k$ that interpolates $u(x)$ at the grid points $x_{j}, j=i-m_{i}, \ldots, i+k-m_{i}-1\left(u\left(x_{j}\right)=S_{i}^{k, m_{i}}\left(x_{j}\right)\right)$. For exponential sums of order $k$, the difference scheme (3.1) is exact:

$$
\mathcal{L} S_{i}^{k, m_{i}}\left(x_{i}\right)=\sum_{j=-m_{i}}^{k-m_{i}-1}\left(\mathcal{L}_{i} L_{j}^{k, m_{i}}\right)(0) S_{i}^{k, m_{i}}\left(x_{i+j}\right)
$$

Further, since $u$ satisfies the differential equation (1.1), i.e., $\mathcal{L} u(x)=f(x), \tau_{i}$ reads

$$
\begin{aligned}
\tau_{i} & =\mathcal{L} S_{i}^{k, m_{i}}\left(x_{i}\right)-\mathcal{L} u\left(x_{i}\right) \\
& =\varepsilon\left(\left(S_{i}^{k, m_{i}}\right)^{\prime \prime}\left(x_{i}\right)-u^{\prime \prime}\left(x_{i}\right)\right)+b_{i}\left(\left(S_{i}^{k, m_{i}}\right)^{\prime}\left(x_{i}\right)-u^{\prime}\left(x_{i}\right)\right) .
\end{aligned}
$$

Now, the approximation error for $u^{\prime}(x)$ is bounded by (cf. Lemma 2.1)

$$
\left|u^{\prime}\left(x_{i}\right)-\left(S_{i}^{k, m_{i}}\right)^{\prime}\left(x_{i}\right)\right| \leq h^{k-2}\left|\rho_{i}\right| C_{k, 1}\left(\rho_{i}, m_{i}\right)\left\|\frac{h}{\rho_{i}} u^{(k)}+u^{(k-1)}\right\|_{\infty}^{k, m_{i}, i},
$$

and the approximation error for $u^{\prime \prime}(x)$ is bounded by

$$
\left|u^{\prime \prime}\left(x_{i}\right)-\left(S_{i}^{k, m_{i}}\right)^{\prime \prime}\left(x_{i}\right)\right| \leq h^{k-3}\left|\rho_{i}\right| C_{k, 2}\left(\rho_{i}, m_{i}\right)\left\|\frac{h}{\rho_{i}} u^{(k)}+u^{(k-1)}\right\|_{\infty}^{k, m_{i}, i}
$$

where

$$
\|f\|_{\infty}^{k, m, i}=\max _{x \in\left[x_{i-m}, x_{i-m+k-1}\right]}|f(x)| .
$$

Note that $S_{i}^{k, m_{i}}$ interpolates $u$ at the nodes $x_{i-m_{i}}, \ldots, x_{i-m_{i}+k-1}$ and that the second argument of the functions $C_{k, r}$ is $\left(x_{i}-x_{i-m_{i}}\right) / h=m_{i}$. Using (4.2) and (4.3) we obtain

$$
\begin{aligned}
\left|\tau_{i}\right| & \leq \varepsilon\left|\left(S_{i}^{k, m_{i}}\right)^{\prime \prime}\left(x_{i}\right)-u^{\prime \prime}\left(x_{i}\right)\right|+\left|b_{i}\right|\left|\left(S_{i}^{k, m_{i}}\right)^{\prime}\left(x_{i}\right)-u^{\prime}\left(x_{i}\right)\right| \\
& \leq\left(h^{k-3} \varepsilon+h^{k-2}\right)\left|\rho_{i}\right| \bar{C}_{k}^{m_{i}}\left(\rho_{i}\right)\left\|\frac{h}{\rho_{i}} u^{(k)}+u^{(k-1)}\right\|_{\infty}^{k, m_{i}, i},
\end{aligned}
$$

where $\bar{C}_{k}^{m}$ is defined by

$$
\bar{C}_{k}^{m}(\rho):=\max \left\{C_{k, 2}(\rho, m),\|b\|_{\infty} C_{k, 1}(\rho, m)\right\} .
$$


Lemma 2.2 and (4.5) imply that

$$
\lim _{\varepsilon \rightarrow 0}\left|\tau_{i}\right| \leq R\left|b_{i}\right| h^{k-2},
$$

for some constant $R$. The previous findings can be summarized in the next theorem.

THEOREM 4.1. Let $n \geq k$ and $k \geq 3$ be arbitrary, and let $u \in C^{k}(0,1)$ be a solution of (1.1)-(1.2). Then for any $i \in\{0, \ldots, n\}$ and $m_{i}\left(0 \leq m_{i} \leq k-1,0 \leq i-m_{i} \leq n-k+1\right)$, the difference scheme defined by (3.1) is consistent, and $\tau_{i}$ defined by (4.1) satisfies

$$
\left|\tau_{i}\right| \leq\left(h^{k-2} \varepsilon+h^{k-1}\right) \bar{C}_{k}^{m_{i}}\left(\rho_{i}\right)\left\|u^{(k)}+\frac{\rho_{i}}{h} u^{(k-1)}\right\|_{\infty}^{k, m_{i}, i}
$$

and

$$
\left|\tau_{i}\right| \leq\left(h^{k-3} \varepsilon+h^{k-2}\right)\left|\rho_{i}\right| \bar{C}_{k}^{m_{i}}\left(\rho_{i}\right)\left\|\frac{h}{\rho_{i}} u^{(k)}+u^{(k-1)}\right\|_{\infty}^{k, m_{i}, i},
$$

where $h=1 / n$. The function $\bar{C}_{k}^{m_{i}}\left(\rho_{i}\right)$ is defined by (4.6). Further, if $b(x) \neq 0$ on $[0,1]$, then there exists a constant $C$, independent on $h$, such that

$$
\lim _{\varepsilon \rightarrow 0}\left|\tau_{i}\right| \leq C h^{k-2},
$$

for $b(x)<0$ and $m_{i}<k-1$, or $b(x)>0$ and $m_{i}>0$.

To study stability (and convergence) of difference schemes, it is more convenient to use a matrix notation. Our goal is to bound the error $u\left(x_{i}\right)-u_{i}$, where $u$ is the exact solution of the problem (1.1)-(1.2). Let us define the vectors

$$
\mathbf{u}=\left[\begin{array}{c}
u\left(x_{0}\right) \\
u\left(x_{1}\right) \\
u\left(x_{2}\right) \\
\vdots \\
u\left(x_{n-1}\right) \\
u\left(x_{n}\right)
\end{array}\right], \quad \mathbf{u}_{\mathbf{n}}=\left[\begin{array}{c}
u_{0} \\
u_{1} \\
u_{2} \\
\vdots \\
u_{n-1} \\
u_{n}
\end{array}\right], \quad \text { and } \quad \mathbf{f}=\left[\begin{array}{c}
\alpha_{0} \\
f_{1} \\
f_{2} \\
\vdots \\
f_{n-1} \\
\alpha_{1}
\end{array}\right]
$$

The equations (3.2) together with the additional $n-1$ equations of the form (3.1) define a system matrix that we denote with $\mathbf{L}_{\mathbf{k}, \mathbf{n}}$, and $\mathbf{u}_{\mathbf{n}}$ is a solution of the equation

$$
\mathbf{L}_{\mathbf{k}, \mathbf{n}} \mathbf{u}_{\mathbf{n}}=\mathbf{f} \text {. }
$$

We define a vector $\tau$ as

$$
\tau=\mathbf{L}_{\mathbf{k}, \mathbf{n}} \mathbf{u}-\mathbf{L}_{\mathbf{k}, \mathbf{n}} \mathbf{u}_{\mathbf{n}}=\mathbf{L}_{\mathbf{k}, \mathbf{n}}\left(\mathbf{u}-\mathbf{u}_{\mathbf{n}}\right) .
$$

If the matrix $\mathbf{L}_{\mathbf{k}, \mathbf{n}}$ is regular, then

$$
\left\|\mathbf{u}-\mathbf{u}_{\mathbf{n}}\right\|_{\infty} \leq\left\|\mathbf{L}_{\mathbf{k}, \mathbf{n}}^{-1}\right\|_{\infty}\|\tau\|_{\infty} .
$$

A bound for $\tau$ is given in Theorem 4.1, and thus the next corollary follows.

COROLLARY 4.2. Let the integers $n \geq k$ and $k \geq 3$ be arbitrary, and let $u \in C^{k}(0,1)$ be a solution of (1.1)-(1.2). If $n-1$ equations of the form (3.1) together with (3.2) define a regular matrix $\mathbf{L}_{\mathbf{k}, \mathbf{n}}$, then $\mathbf{u}_{\mathbf{n}}=\left[u_{0}, \ldots, u_{n}\right]^{T}$, a solution of (4.7), satisfies

$$
\left|u_{j}-u\left(x_{j}\right)\right| \leq\left\|\mathbf{L}_{\mathbf{k}, \mathbf{n}}^{-\mathbf{1}}\right\|_{\infty}\left(h^{k-2} \varepsilon+h^{k-1}\right) \max _{i} \bar{C}_{k}^{m_{i}}\left(\rho_{i}\right)\left\|u^{(k)}+\frac{\rho_{i}}{h} u^{(k-1)}\right\|_{\infty}^{k, m_{i}, i}
$$


and

$$
\left|u_{j}-u\left(x_{j}\right)\right| \leq\left\|\mathbf{L}_{\mathbf{k}, \mathbf{n}}^{-\mathbf{1}}\right\|_{\infty}\left(h^{k-3} \varepsilon+h^{k-2}\right) \max _{i}\left|\rho_{i}\right| \bar{C}_{k}^{m_{i}}\left(\rho_{i}\right)\left\|\frac{h}{\rho_{i}} u^{(k)}+u^{(k-1)}\right\|_{\infty}^{k, m_{i}, i},
$$

where $h=1 / n$ and $j=0,1, \ldots, n$. If $b(x) \neq 0$ on $[0,1]$, then there exists a constant $C$, independent on $h$, such that

$$
\lim _{\varepsilon \rightarrow 0}\left|u_{j}-u\left(x_{j}\right)\right| \leq C h^{k-2} \lim _{\varepsilon \rightarrow 0}\left\|\mathbf{L}_{\mathbf{k}, \mathbf{n}}^{-\mathbf{1}}\right\|_{\infty},
$$

if the limit $\lim _{\varepsilon \rightarrow 0}\left\|\mathbf{L}_{\mathbf{k}, \mathbf{n}}^{-1}\right\|_{\infty}$ exists and when $b(x)<0$ and $m_{i}<k-1$ or $b(x)>0$ and $m_{i}>0$.

There is no general approach to bound $\mathbf{L}_{\mathbf{k}, \mathbf{n}}^{-1}$. Instead, we have to consider each method separately.

5. Three-point difference schemes. Here we exemplify our approach in the simplest case, the three-point difference schemes. In the analysis of stability (and convergence), in addition to assumptions (1.3), we assume that the coefficient $c$ satisfies

$$
-c(x) \geq c_{\min }>0 \quad \text { for } x \in[0,1] .
$$

Further, the coefficient $b$ is restricted to $b(x) \neq 0$ for all $x$, i.e., we exclude the so-called turning point case. We propose three three-point difference schemes $(k=3)$ with a uniform choice of $m_{i}, m_{i}=m, m=0,1,2$.

The system matrix (4.7), here denoted by $\mathbf{L}_{\mathbf{3}, \mathbf{m}, \mathbf{n}}$, is a tridiagonal matrix

$$
\mathbf{L}_{\mathbf{3}, \mathbf{m}, \mathbf{n}}=\left[\begin{array}{cccccc}
1 & & & & & \mathbf{0} \\
r_{m}^{m} & s_{m}^{m} & t_{m}^{m} & & & \\
& r_{1+m}^{m} & s_{1+m}^{m} & t_{1+m}^{m} & & \\
& & \ddots & \ddots & \ddots & \\
& & & r_{n-2+m}^{m} & s_{n-2+m}^{m} & t_{n-2+m}^{m} \\
\mathbf{0} & & & & & 1
\end{array}\right]
$$

Since the approximation satisfies the boundary conditions, it holds that $\tau_{0}=0$ and $\tau_{n}=0$. Therefore, we may omit the first and the last equation from the system, and we can consider the simplified matrix

$$
\mathbf{L}_{\mathbf{3}, \mathbf{m}, \mathbf{n}}=\left[\begin{array}{ccccc}
s_{m}^{m} & t_{m}^{m} & & & \mathbf{0} \\
r_{1+m}^{m} & s_{1+m}^{m} & t_{1+m}^{m} & & \\
& \ddots & \ddots & \ddots & \\
& & r_{n-3+m}^{m} & s_{n-3+m}^{m} & t_{n-3+m}^{m} \\
\mathbf{0} & & & r_{n-2+m}^{m} & s_{n-2+m}^{m}
\end{array}\right] .
$$

Theorem 4.1 states that the methods are consistent. But, to prove convergence of the methods we have to consider stability, i.e., boundedness of the matrix $\mathbf{L}_{\mathbf{3}, \mathbf{m}, \mathbf{n}}^{\mathbf{- 1}}$. This will be done in the forthcoming section.

5.1. A difference scheme for $\boldsymbol{m}=\mathbf{0}$. The general scheme (3.1) for $k=3$ and $m=0$ reads:

$$
\left(\mathcal{L}_{i} L_{0}^{3,0}\right)(0) u_{i}+\left(\mathcal{L}_{i} L_{1}^{3,0}\right)(0) u_{i+1}+\left(\mathcal{L}_{i} L_{2}^{3,0}\right)(0) u_{i+2}=f\left(x_{i}\right), \quad i=0, \ldots, n-2,
$$


with the end conditions $u_{0}=\alpha_{0}$ and $u_{n}=\alpha_{1}$. A straightforward calculation results in the coefficients

$$
\begin{aligned}
r_{i}^{0} & =\left(\mathcal{L}_{i} L_{0}^{3,0}\right)(0)=c_{i}+\frac{b_{i}}{h} \frac{1}{\mathrm{e}^{\rho_{i}}-1}+\frac{\rho_{i}}{h} \frac{\mathrm{e}^{2 \rho_{i}}}{\left(\mathrm{e}^{\rho_{i}}-1\right)^{2}}\left(\frac{\varepsilon \rho_{i}}{h}-b_{i}\right), \\
s_{i}^{0} & =\left(\mathcal{L}_{i} L_{1}^{3,0}\right)(0)=-\frac{b_{i}}{h} \frac{\mathrm{e}^{\rho_{i}}+1}{\mathrm{e}^{\rho_{i}}-1}-\frac{\rho_{i}}{h} \frac{2 \mathrm{e}^{2 \rho_{i}}}{\left(\mathrm{e}^{\rho_{i}}-1\right)^{2}}\left(\frac{\varepsilon \rho_{i}}{h}-b_{i}\right), \\
t_{i}^{0} & =\left(\mathcal{L}_{i} L_{2}^{3,0}\right)(0)=\frac{b_{i}}{h} \frac{\mathrm{e}^{\rho_{i}}}{\mathrm{e}^{\rho_{i}}-1}+\frac{\rho_{i}}{h} \frac{\mathrm{e}^{2 \rho_{i}}}{\left(\mathrm{e}^{\rho_{i}}-1\right)^{2}}\left(\frac{\varepsilon \rho_{i}}{h}-b_{i}\right),
\end{aligned}
$$

for $i=0, \ldots, n-2$.

LEMMA 5.1. Let $-c(x) \geq c_{\min }>0$ and $b(x)>0$ for $x \in[0,1]$. If the $\rho_{i}$ 's are chosen according to (3.4), then the matrix $\mathbf{L}_{\mathbf{3}, \mathbf{0}, \mathbf{n}}$ is diagonally dominant, and it holds that

$$
\left\|\mathbf{L}_{\mathbf{3}, \mathbf{0}, \mathbf{n}}^{-\mathbf{1}}\right\|_{\infty} \leq \frac{1}{c_{\min }}
$$

Proof. First, note that from (3.3) it easily follows that

$$
\frac{\varepsilon \rho_{i}}{h}-b_{i}=-\frac{c_{i} h}{\rho_{i}} \geq 0 .
$$

Therefore, the entries of the matrix $\mathbf{L}_{\mathbf{3}, \mathbf{0}, \mathbf{n}}$ satisfy

$$
s_{i}^{0} \leq 0 \quad \text { and } \quad t_{i}^{0} \geq 0 .
$$

The coefficient $r_{i}^{0}$ is given by

$$
r_{i}^{0}=c_{i}+\frac{b_{i}}{h} \frac{1}{\mathrm{e}^{\rho_{i}}-1}-c_{i} \frac{\mathrm{e}^{2 \rho_{i}}}{\left(\mathrm{e}^{\rho_{i}}-1\right)^{2}} \geq c_{i}-c_{i} \frac{\mathrm{e}^{2 \rho_{i}}}{\left(\mathrm{e}^{\rho_{i}}-1\right)^{2}}=-c_{i}\left(\frac{\mathrm{e}^{2 \rho_{i}}}{\left(\mathrm{e}^{\rho_{i}}-1\right)^{2}}-1\right) .
$$

This inequality holds because of the positive signs of $b$ and $\rho_{i}$. Since

$$
\frac{\mathrm{e}^{2 \rho_{i}}}{\left(\mathrm{e}^{\rho_{i}}-1\right)^{2}}=\frac{1}{\left(1-\mathrm{e}^{-\rho_{i}}\right)^{2}} \geq 1
$$

it follows that

$$
r_{i}^{0} \geq 0
$$

Now we easily obtain that

$$
\left|s_{i}^{0}\right|-\left|r_{i}^{0}\right|-\left|t_{i}^{0}\right|=-s_{i}^{0}-r_{i}^{0}-t_{i}^{0}=-c_{i} \geq c_{\min }>0,
$$

and the bound from Lemma 5.1 is proved.

One may note that for the choice of the $\rho_{i}$ 's according to (3.5), $r_{i}^{0}$ is positive for small $\rho_{i}$ and negative for large $\rho_{i}$. Thus, a simple argument of diagonal dominance does not apply in this case.

5.2. A difference scheme for $m=1$. The central-point scheme is given by

$$
\left(\mathcal{L}_{i} L_{-1}^{3,1}\right)(0) u_{i-1}+\left(\mathcal{L}_{i} L_{0}^{3,1}\right)(0) u_{i}+\left(\mathcal{L}_{i} L_{1}^{3,1}\right)(0) u_{i+1}=f\left(x_{i}\right),
$$


$i=1, \ldots, n-1$, with two additional equations obtained from the boundary conditions $u_{0}=\alpha_{0}$ and $u_{n}=\alpha_{1}$. The explicit expressions for the coefficients of the scheme (5.1) are

$$
\begin{aligned}
& r_{i}^{1}=\left(\mathcal{L}_{i} L_{-1}^{3,1}\right)(0)=\frac{\varepsilon \mathrm{e}^{\rho_{i}} \rho_{i}^{2}}{\left(\mathrm{e}^{\rho_{i}}-1\right)^{2} h^{2}}+b_{i} \frac{-1-\mathrm{e}^{\rho_{i}}\left(\rho_{i}-1\right)}{\left(\mathrm{e}^{\rho_{i}}-1\right)^{2} h}, \\
& s_{i}^{1}=\left(\mathcal{L}_{i} L_{0}^{3,1}\right)(0)=\frac{-2 \varepsilon \mathrm{e}^{\rho_{i}} \rho_{i}^{2}}{\left(\mathrm{e}^{\rho_{i}}-1\right)^{2} h^{2}}+b_{i} \frac{1-\mathrm{e}^{2 \rho_{i}}+2 \rho_{i} \mathrm{e}^{\rho_{i}}}{\left(\mathrm{e}^{\rho_{i}}-1\right)^{2} h}+c_{i}, \\
& t_{i}^{1}=\left(\mathcal{L}_{i} L_{1}^{3,1}\right)(0)=\frac{\varepsilon \mathrm{e}^{\rho_{i}} \rho_{i}^{2}}{\left(\mathrm{e}^{\rho_{i}}-1\right)^{2} h^{2}}+b_{i} \frac{\mathrm{e}^{\rho_{i}}\left(\mathrm{e}^{\rho_{i}}-\rho_{i}-1\right)}{\left(\mathrm{e}^{\rho_{i}}-1\right)^{2} h},
\end{aligned}
$$

for $i=1, \ldots, n-1$. The parameter $\rho_{i}$ is defined either by (3.4) or (3.5). A bound for the matrix $\mathbf{L}_{\mathbf{3}, \mathbf{1}, \mathbf{n}}{ }^{-1}$ is given in the following lemma.

LEMMA 5.2. Let $-c(x) \geq c_{\text {min }}>0$ and $b(x) \neq 0$ for $x \in[0,1]$. If the $\rho_{i}$ 's are chosen according to (3.4) or (3.5), then the matrix $\mathbf{L}_{\mathbf{3}, \mathbf{1}, \mathbf{n}}$ is diagonally dominant, and

$$
\left\|\mathbf{L}_{\mathbf{3}, \mathbf{1}, \mathbf{n}}^{-\mathbf{1}}\right\|_{\infty} \leq \frac{1}{c_{\min }}
$$

Proof. First, we prove that the coefficients $s_{i}^{1}, r_{i}^{1}$, and $t_{i}^{1}, i=1, \ldots, n-1$, satisfy

$$
s_{i}^{1}<0 \quad \text { and } \quad t_{i}^{1}, r_{i}^{1}>0 .
$$

From the definition of $\rho_{i}(3.4)$, we have

$$
\frac{\varepsilon}{h^{2}} \rho_{i}^{2}-\frac{b_{i}}{h} \rho_{i}+c_{i}=0 .
$$

Hence,

$$
\frac{\varepsilon}{h} \rho_{i}^{2}=b_{i} \rho_{i}-c_{i} h>b_{i} \rho_{i}, \quad i=1, \ldots, n-1 .
$$

If $\rho_{i}$ is defined according to (3.5), then

$$
\frac{\varepsilon}{h} \rho_{i}^{2}=b_{i} \rho_{i}, \quad i=1, \ldots, n-1 .
$$

For $r_{i}^{1}$ we have, using (5.2) or (5.3),

$$
\begin{aligned}
r_{i}^{1} & =\frac{\varepsilon \mathrm{e}^{\rho_{i}} \rho_{i}^{2}}{\left(\mathrm{e}^{\rho_{i}}-1\right)^{2} h^{2}}-b_{i} \frac{1+\mathrm{e}^{\rho_{i}}\left(\rho_{i}-1\right)}{\left(\mathrm{e}^{\rho_{i}}-1\right)^{2} h}=\frac{\frac{\varepsilon \mathrm{e}^{\rho_{i}} \rho_{i}^{2}}{h}-b_{i}\left(1+\mathrm{e}^{\rho_{i}}\left(\rho_{i}-1\right)\right)}{\left(\mathrm{e}^{\rho_{i}}-1\right)^{2} h} \\
& \geq \frac{b_{i}\left(\rho_{i} \mathrm{e}^{\rho_{i}}-\left(1+\rho_{i} \mathrm{e}^{\rho_{i}}-\mathrm{e}^{\rho_{i}}\right)\right)}{\left(\mathrm{e}^{\rho_{i}}-1\right)^{2} h}=\frac{b_{i}}{\left(\mathrm{e}^{\rho_{i}}-1\right) h}>0
\end{aligned}
$$

because $b_{i}$ and $\rho_{i}$ (and therefore $b_{i}$ and $\mathrm{e}^{\rho_{i}}-1$ ) have the same sign. For $t_{i}$ we have, using (5.2) or (5.3), that

$$
\begin{aligned}
t_{i}^{1} & =\frac{\varepsilon \mathrm{e}^{\rho_{i}} \rho_{i}^{2}}{\left(\mathrm{e}^{\rho_{i}}-1\right)^{2} h^{2}}+b_{i} \frac{\mathrm{e}^{\rho_{i}}\left(\mathrm{e}^{\rho_{i}}-\rho_{i}-1\right)}{\left(\mathrm{e}^{\rho_{i}}-1\right)^{2} h}=\frac{\left(\frac{\varepsilon \mathrm{e}^{\rho_{i}} \rho_{i}^{2}}{h}+b_{i} \mathrm{e}^{\rho_{i}}\left(\mathrm{e}^{\rho_{i}}-\rho_{i}-1\right)\right)}{\left(\mathrm{e}^{\rho_{i}}-1\right)^{2} h} \\
& >\frac{b_{i} \mathrm{e}^{\rho_{i}}}{\left(\mathrm{e}^{\rho_{i}}-1\right)^{2} h}\left(\rho_{i}+\mathrm{e}^{\rho_{i}}-\rho_{i}-1\right)=\frac{b_{i} \mathrm{e}^{\rho_{i}}}{\left(\mathrm{e}^{\rho_{i}}-1\right) h}>0,
\end{aligned}
$$


where we again used that $\rho_{i}$ and $b_{i}$ have the same sign. From the definition of $r_{i}, s_{i}$, and $t_{i}$ it is clear that $s_{i}+r_{i}+t_{i}=c_{i}$, and

$$
s_{i}^{1}=c_{i}-r_{i}^{1}-t_{i}^{1}<0 .
$$

Since

$$
\left|s_{i}^{1}\right|-\left|r_{i}^{1}\right|-\left|t_{i}^{1}\right|=-s_{i}^{1}-r_{i}^{1}-t_{i}^{1}=-c_{i} \geq c_{\min }>0,
$$

we conclude that $\mathbf{L}_{\mathbf{3}, \mathbf{1}, \mathbf{n}}$ is diagonally dominant, and

$$
\left\|\mathbf{L}_{\mathbf{3}, \mathbf{1}, \mathbf{n}}^{-\mathbf{1}}\right\|_{\infty} \leq \frac{1}{c_{\min }}
$$

5.3. A difference scheme for $\boldsymbol{m}=\mathbf{2}$. When $m=2$, the three-point scheme reads

$$
\left(\mathcal{L}_{i} L_{-2}^{3,2}\right)(0) u_{i-2}+\left(\mathcal{L}_{i} L_{-1}^{3,2}\right)(0) u_{i-1}+\left(\mathcal{L}_{i} L_{0}^{3,2}\right)(0) u_{i}=f\left(x_{i}\right), \quad i=2, \ldots, n
$$

with the end conditions $u_{0}=\alpha_{0}$ and $u_{n}=\alpha_{1}$. The coefficients from the scheme (5.4) are

$$
\begin{aligned}
r_{i}^{2} & =\left(\mathcal{L}_{i} L_{-2}^{3,2}\right)(0)=\frac{\varepsilon}{h^{2}} \frac{\rho_{i}^{2}}{\left(\mathrm{e}^{\rho_{i}}-1\right)^{2}}+\frac{b_{i}}{h} \frac{-1+\mathrm{e}^{\rho_{i}}-\rho_{i}}{\left(\mathrm{e}^{\rho_{i}}-1\right)^{2}}, \\
s_{i}^{2} & =\left(\mathcal{L}_{i} L_{-1}^{3,2}\right)(0)=\frac{\varepsilon}{h^{2}} \frac{-2 \rho_{i}^{2}}{\left(\mathrm{e}^{\rho_{i}}-1\right)^{2}}+\frac{b_{i}}{h} \frac{1+2 \rho_{i}-\mathrm{e}^{2 \rho_{i}}}{\left(\mathrm{e}^{\rho_{i}}-1\right)^{2}}, \\
t_{i}^{2} & =\left(\mathcal{L}_{i} L_{0}^{3,2}\right)(0)=\frac{\varepsilon}{h^{2}} \frac{\rho_{i}^{2}}{\left(\mathrm{e}^{\rho_{i}}-1\right)^{2}}+\frac{b_{i}}{h} \frac{\mathrm{e}^{2 \rho_{i}}-\mathrm{e}^{\rho_{i}}-\rho_{i}}{\left(\mathrm{e}^{\rho_{i}}-1\right)^{2}}+c_{i},
\end{aligned}
$$

for $i=2, \ldots, n$.

LEMMA 5.3. Let $-c(x) \geq c_{\min }>0$ and $b(x)>0$ for $x \in[0,1]$. If the $\rho_{i}$ 's are chosen according to (3.4) or (3.5), then the matrix $\mathbf{L}_{\mathbf{3 , 2}, \mathbf{n}}$ is diagonally dominant, and

$$
\left\|\mathbf{L}_{\mathbf{3}, \mathbf{2}, \mathbf{n}}^{-\mathbf{1}}\right\|_{\infty} \leq \frac{1}{c_{\min }}
$$

for $h=1 / n$ satisfying

$$
h \leq \min _{x \in[0,1]}\left|\frac{b(x)}{c(x)}\right| .
$$

Proof. Because of

$$
\mathrm{e}^{\rho_{i}}-1-\rho_{i} \geq 0 \quad \text { and } \quad \mathrm{e}^{2 \rho_{i}}-1-2 \rho_{i} \geq 0
$$

for $\rho_{i} \geq 0$, the coefficients $r_{i}^{2}$ and $s_{i}^{2}$ satisfy

$$
r_{i}^{2} \geq 0 \quad \text { and } \quad s_{i}^{2} \leq 0 .
$$

Also, for positive $\rho_{i}$,

$$
\frac{\mathrm{e}^{2 \rho_{i}}-\mathrm{e}^{\rho_{i}}-\rho_{i}}{\left(\mathrm{e}^{\rho_{i}}-1\right)^{2}} \geq 1
$$


Now,

$$
t_{i}^{2} \geq \frac{b_{i}}{h} \frac{\mathrm{e}^{2 \rho_{i}}-\mathrm{e}^{\rho_{i}}-\rho_{i}}{\left(\mathrm{e}^{\rho_{i}}-1\right)^{2}}+c_{i} \geq \frac{b_{i}}{h}+c_{i} \geq 0 .
$$

The last inequality follows from the condition (5.5). As in the previous cases,

$$
\left|s_{i}^{2}\right|-\left|r_{i}^{2}\right|-\left|t_{i}^{2}\right|=-s_{i}^{2}-r_{i}^{2}-t_{i}^{2}=-c_{i} \geq c_{\min }>0,
$$

which proves the diagonal dominance and the bound of the lemma.

We summarize all previous results in the following theorem.

THEOREM 5.4. Let $u \in C^{3}(0,1)$ be the solution of (1.1)-(1.2), where $-c(x) \geq c_{\min }>0$ and $b(x) \neq 0$ on $[0,1]$. Let $\mathbf{u}_{\mathbf{n}}=\left[u_{0}, \ldots, u_{n}\right]^{T}$ denote a solution of the difference scheme (4.7), for $k=3, m \in\{0,1,2\}$, and $h=1 / n$. In addition, let the $\rho_{i}$ 's be chosen according to (3.4), and let h satisfy

$$
h \leq \max _{x \in[0,1]}\left|\frac{c(x)}{b(x)}\right|
$$

in the cases $m=0$ for $b<0$, and $m=2$ for $b>0$. Then, the error at the nodes $x_{j}$, $j=0, \ldots, n$, is bounded by

$$
\left|u_{j}-u\left(x_{j}\right)\right| \leq\left(h \varepsilon+h^{2}\right) c_{\min }^{-1} \max _{i} \bar{C}_{3}^{m}\left(\rho_{i}\right)\left\|u^{(3)}+\frac{\rho_{i}}{h} u^{(2)}\right\|_{\infty}^{3, m, i}
$$

and

$$
\left|u_{j}-u\left(x_{j}\right)\right| \leq(\varepsilon+h) c_{\min }^{-1} \max _{i}\left|\rho_{i}\right| \bar{C}_{3}^{m}\left(\rho_{i}\right)\left\|\frac{h}{\rho_{i}} u^{(3)}+u^{(2)}\right\|_{\infty}^{3, m, i} .
$$

The function $\bar{C}_{3}^{m}$ is defined by (4.6), while the norm \|\|$_{\infty}^{3, m, i}$ is defined by (4.4). Further, there exists a constant $C$, independent of $h$, such that

$$
\lim _{\varepsilon \rightarrow 0}\left|u_{j}-u\left(x_{j}\right)\right| \leq C h,
$$

when $b<0$ and $m<2$ or $b>0$ and $m>0$.

Proof. In the case $m=1$, the assertion of the theorem follows directly from Corollary 4.2 by a direct substitution of $\left\|\mathbf{L}_{\mathbf{3}, \mathbf{1}, \mathbf{n}}^{-1}\right\|_{\infty}$ with the bound from Lemma 5.2.

Bounds for $\left\|\mathbf{L}_{\mathbf{3}, \mathbf{2}, \mathbf{n}}^{-\mathbf{1}}\right\|_{\infty}$ and $\left\|\mathbf{L}_{\mathbf{3}, \mathbf{0}, \mathbf{n}}^{-\mathbf{1}}\right\|_{\infty}$ are given by Lemma 5.3 and Lemma 5.1 in the case when $b(x)>0$. When $b(x)<0$, the simple substitution $t=1-x$ transforms the problem (1.1)-(1.2) into the case $-b(x)>0$. With such a substitution, the scheme for $m=0$ becomes the scheme for $m=2$ and vice versa. We may also verify that substituting $\rho_{i}$ into $-\rho_{i}$ and $b_{i}$ into $-b_{i}$ transforms the scheme $m=2$ into the scheme for $m=0$, as well as the scheme $m=0$ into the scheme for $m=2$. (Note that for $b_{i}>0$ the parameter $\rho_{i}$ is positive, while for $b_{i}<0$ the parameter $\rho_{i}$ is negative.) Thus, $\left\|\mathbf{L}_{\mathbf{3}, \mathbf{2}, \mathbf{n}}^{-\mathbf{1}}\right\|_{\infty}$ and $\left\|\mathbf{L}_{\mathbf{3}, \mathbf{0}, \mathbf{n}}^{-\mathbf{1}}\right\|_{\infty}$ are bounded for $b(x) \neq 0$, and the error estimate follows directly from Corollary 4.2 .

Theorem 5.4 may be applied to the choice of $\rho_{i}$ 's given by (3.5) with the exception of the cases $m=0$ for $b>0$ and $m=2$ for $b<0$.

6. Numerical examples. In this section we illustrate our methods with examples having a known analytical expression for the exact solution. 


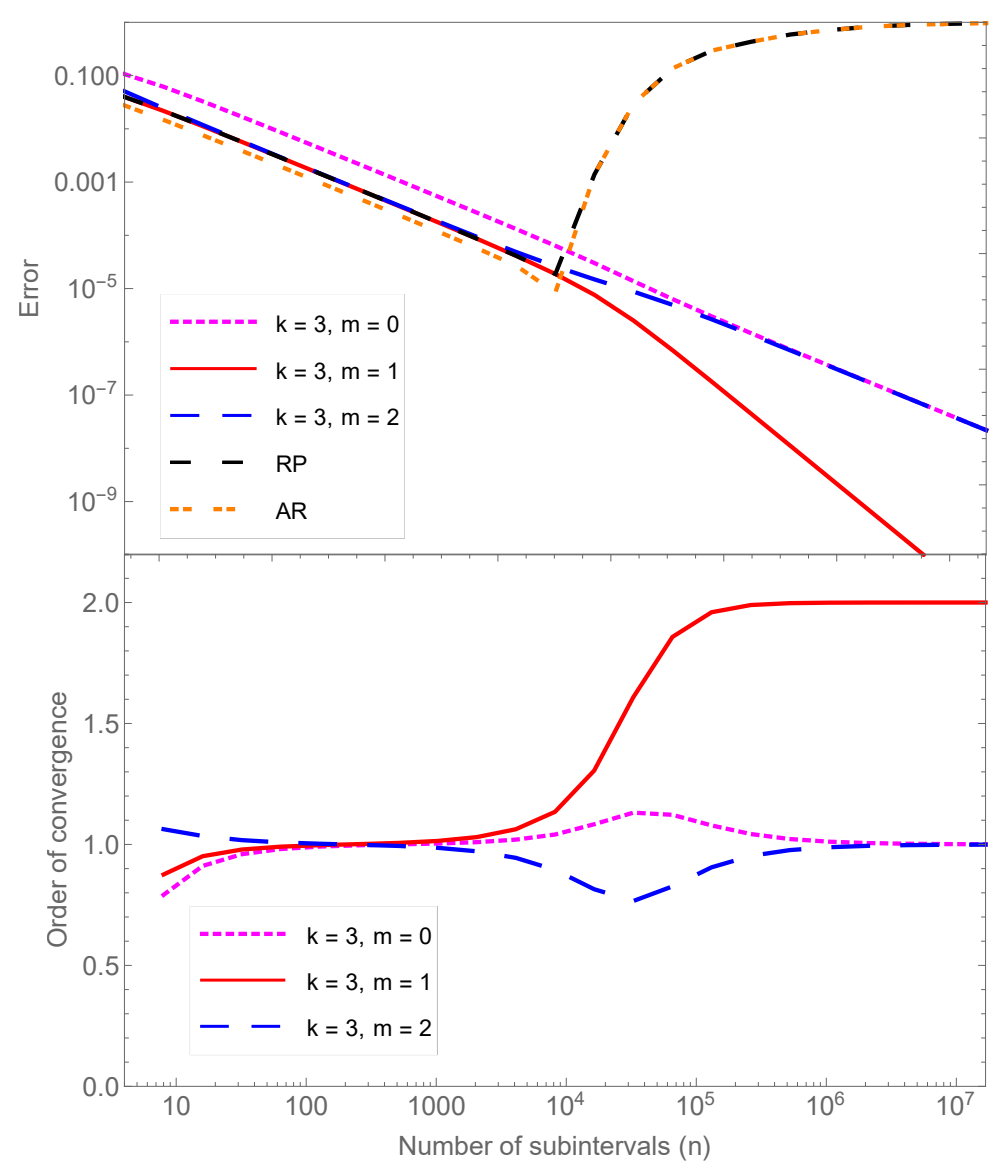

FIG. 6.1. Error for the solution of (6.1)-(6.2) and the numerical order of convergence by different methods in dependence of the number of subintervals for a perturbation parameter $\varepsilon=10^{-5}$.

EXAMPLE 6.1. Consider:

$$
\varepsilon y^{\prime \prime}+y^{\prime}-(1+\varepsilon) y=0,
$$

$$
y(0)=1+\mathrm{e}^{-1}, \quad y(1)=1+\mathrm{e}^{-\frac{1+\varepsilon}{\varepsilon}} .
$$

The exact solution of problem (6.1)-(6.2) is

$$
y(x)=\mathrm{e}^{-x \frac{1+\varepsilon}{\varepsilon}}+\mathrm{e}^{x-1} .
$$

We apply difference schemes to this problem using $2^{n}$ subintervals, for $n=2, \ldots, 24$. The perturbation parameter $\varepsilon$ is set to $10^{-5}$. The maximum error at the grid points in dependency on the number of subintervals is shown in Figure 6.1.

As could be expected, the central point scheme $(m=1)$ gives the best approximation. Further, we compare our methods to those proposed by Reddy and Chakravarthy [14] (denoted by RP) and Awoke and Reddy [1] (denoted by AR). Both methods are exponentially fitted, 


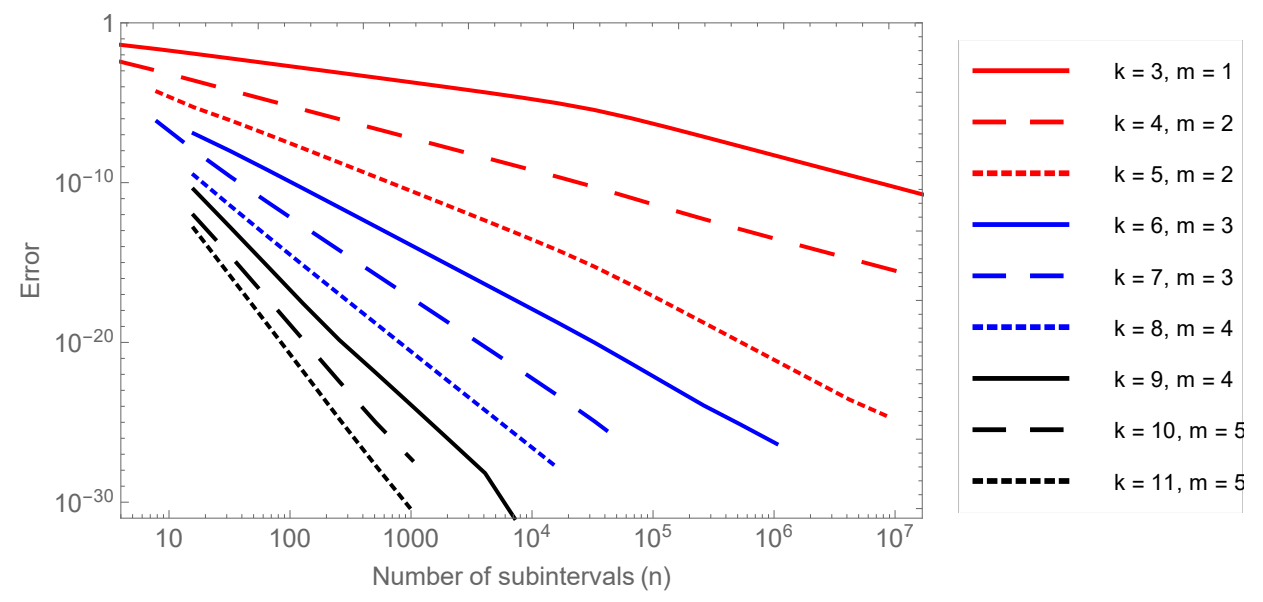

FIG. 6.2. Error for the solution of (6.1)-(6.2) by the central difference methods of different order in dependence of the number of subintervals for a perturbation parameter $\varepsilon=10^{-5}$.

and the AR method is an improvement of the RP method. When $\varepsilon$ is smaller than the mesh width $h$, the AR method is slightly better for this example than our method for $m=1$, while the RP method gives a solution that is indistinguishable to our method. But both methods (AR and RP) fail to converge to the solution when $h \rightarrow 0$. This lack of convergence is not observed in some other examples (not shown here). If the inner solution is an exponential function, then the proposed schemes give the exact solution since the formulae used for the first and second derivative are exact for exponential functions. Therefore, the approximation error decreases in the case $h<4(k-1) \varepsilon \ln (1 / \varepsilon) / b_{\min }$, too. The difference schemes denoted by AR [1] and RP [14] are not exact for exponential functions and this may be the reason for their behavior.

In Figure 6.1 we also present the numerical order of convergence $\left(r_{n}\right)$. It is calculated according to

$$
r_{n}:=\frac{\ln \left(e_{n-1} / e_{n}\right)}{\ln 2},
$$

where $e_{n}$ is error for the partition with $2^{n}$ subintervals. For $\varepsilon<h$, the convergence of the methods is linear as was shown in Theorem 5.4. More precisely, for small $\varepsilon$, the error is proportional to $\varepsilon+h$. In this case, $\varepsilon$ may be neglected and the convergence is linear.

The same theorem also provides a bound for relatively large $\varepsilon$. Now, the error is proportional to $\varepsilon h+h^{2}$, i.e., the convergence is at least linear. From Figure 6.1, it is evident that when $h$ is relatively small with respect to $\varepsilon$, then the method with $m=1$ converges quadratically. An analysis of the method in the case $h \approx 0$ may explain this behavior.

In this example we used the choice of $\rho_{i}$ given by (3.4). We also tried the choice in (3.5), but there was no difference in the results.

Further, we illustrate some properties of the proposed methods that are not discussed in this paper. First, we applied high-order methods to the problem (6.1)-(6.2). All the used methods appeared to be convergent (Figure 6.2). The order of convergence is $k-2$ for the $k$-point scheme.

EXAMPLE 6.2. An important property of methods for singularly perturbed problems is the independence of the convergence of the perturbation parameter $\varepsilon$. Such convergence is called $\varepsilon$-uniform convergence. A method is $\varepsilon$-uniformly convergent if there exists constants $C$ and $m$, independent of $\varepsilon$, such that the solution $u$ of the problem (1.1)-(1.2) and its approximation 


\section{ETNA}

Kent State University and

Johann Radon Institute (RICAM)

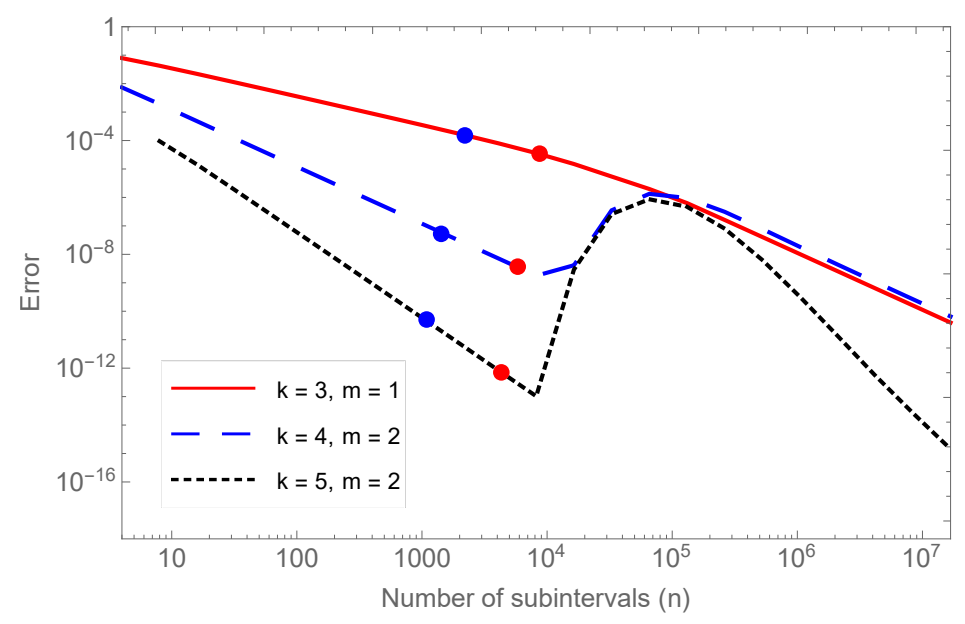

FIG. 6.3. Error for the solution of problem (6.4)-(6.5) for three-, four-, and five-point methods in dependence of the number of subintervals for a perturbation parameter $\varepsilon=10^{-5}$. Blue and red points indicate the error for $h=4(k-1) \varepsilon \ln (1 / \varepsilon) / b_{\min }$ and $h=(k-1) \varepsilon \ln (1 / \varepsilon) / b_{\min }$, respectively.

at the mesh points $u_{i}$ satisfy

$$
\max _{\varepsilon \in[0,1]}\left|u\left(x_{i}\right)-u_{i}\right| \leq C h^{m}
$$

for all $i$.

Although Example 6.1 suggests $\varepsilon$-uniform convergence of the proposed methods, it is known that exponentially fitted methods are not $\varepsilon$-uniformly convergent [16]. In [11] we analyzed the interpolation error by exponential sums for the solution of singularly perturbed boundary value problems, and we demonstrated that the interpolation error is not independent of $\varepsilon$.

Here we consider the equation [11]

$$
\varepsilon u^{\prime \prime}+2(1-x) u^{\prime}-2 u=(\varepsilon-2 x) \mathrm{e}^{x},
$$

with boundary conditions

$$
u(0)=2 \quad \text { and } \quad u(1)=\mathrm{e}^{-1 / \varepsilon}+\mathrm{e} .
$$

The exact solution is given by

$$
u(x)=\mathrm{e}^{-\left(2 x-x^{2}\right) / \varepsilon}+\mathrm{e}^{x} .
$$

This solution has a boundary layer near the point $x=0$.

The dependence of the error on the number of intervals for three-, four-, and five-point methods is presented in Figure 6.3. Figure 6.3 clearly demonstrates that the proposed methods are not $\varepsilon$-uniformly convergent. For $h \geq 4(k-1) \varepsilon \ln (1 / \varepsilon) / b_{\min }$ the behavior of the error is described by Lemma 2.2 (i.e., Theorem 4.1): $\left\|u\left(x_{i}\right)-u_{i}\right\| \leq C_{k} h^{k-2}$ (blue points in the figure). Experimental results in [11] and the figure suggest that the condition (2.7) can be extended to $h \geq(k-1) \varepsilon \ln (1 / \varepsilon) / b_{\min }$ (red points in the figure). After that, the error starts to increase (except for the three-point method). For $h \leq 10^{-5}=\varepsilon$ the error again decreases, which is in accordance with the bound from Theorem 4.1 (bound for 'large' $\rho$ ). Although 


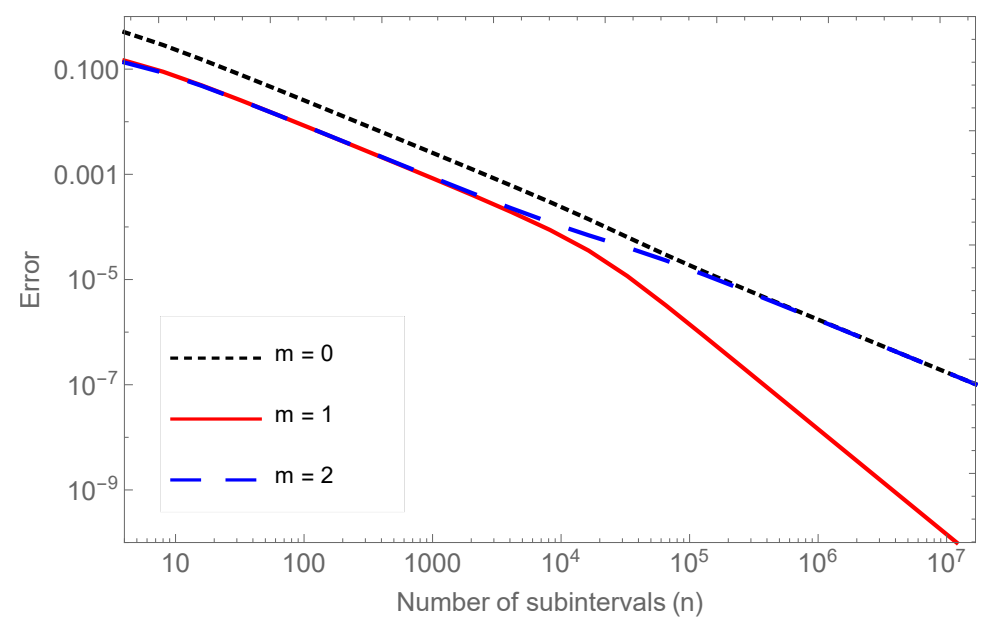

FIG. 6.4. Error for the solution of the advection-diffusion problem (6.6)-(6.7) for the three-point methods in dependence of number of subintervals for a perturbation parameter $\varepsilon=10^{-5}$.

the error for the three-point method seems not to be affected by $\varepsilon$, in [11] we have shown that the interpolation error is not $\varepsilon$-uniform convergent for three-point methods. Numerical experiments from [11] suggest that an implementation of a dense mesh in the boundary layer results in $\varepsilon$-uniform convergence.

EXAMPLE 6.3. Next, we applied the methods to the advection-diffusion problem [8]

$$
\varepsilon y^{\prime \prime}+y^{\prime}=-\mathrm{e}^{1-x}
$$

with the exact solution

$$
y(x)=\left[\mathrm{e}^{1-x}-\frac{1-\mathrm{e}^{1-1 / \varepsilon}+(\mathrm{e}-1) \mathrm{e}^{-x / \varepsilon}}{1-\mathrm{e}^{-1 / \varepsilon}}\right] \frac{1}{1-\varepsilon} .
$$

The dependence of the error on the number of intervals is displayed in Figure 6.4 (for $\varepsilon=10^{-5}$ ). The behavior of the methods is the same as in the case $c(x) \neq 0$ (problem (6.1)-(6.2), Figure 6.1). The convergence of the methods is linear for $h>\varepsilon$, which is in agreement with the theoretical results. The order of the convergence is the same in the case $h<\varepsilon$ for $m=0$ and $m=2$. The method defined by $m=1$ converges quadratically for small values of $h$.

EXAMPLE 6.4. In the last example we demonstrate the applicability of the proposed schemes to nonlinear problems. The problem

$$
\varepsilon y^{\prime \prime}+2 y^{\prime}-\mathrm{e}^{y}=f(x),
$$

with homogeneous boundary conditions $y(0)=0, y(1)=0$, and $f \equiv 0$ has an approximate asymptotic solution

$$
y(x)=\log \frac{2}{3-x}-\log \frac{2}{3} \mathrm{e}^{-2 x / \varepsilon} .
$$




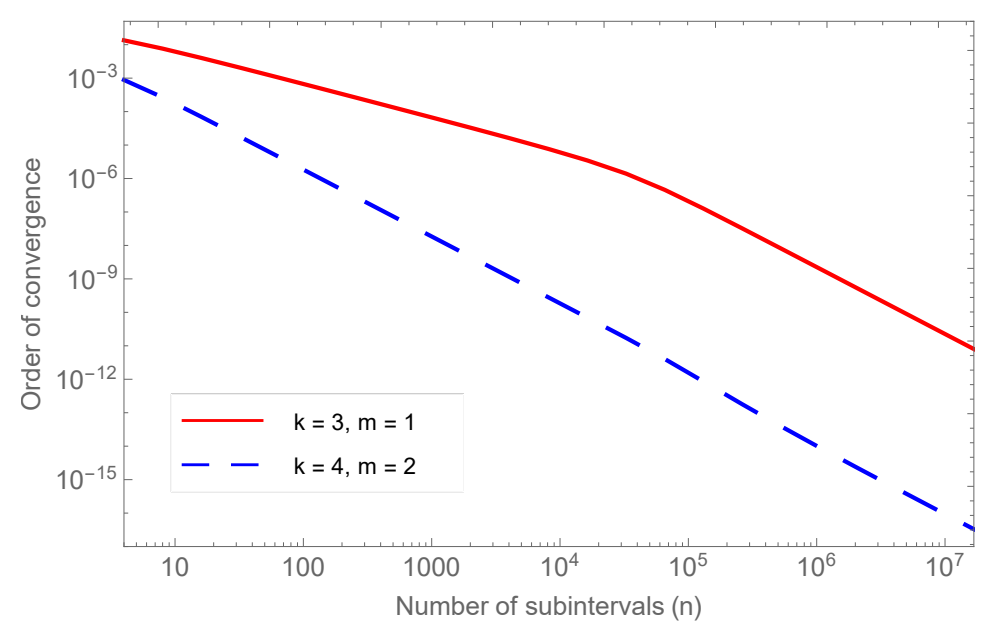

FIG. 6.5. Error for the solution of nonlinear problem (6.8) for three-point and four-point method in dependence of the number of subintervals for a perturbation parameter $\varepsilon=10^{-5}$.

In the example we impose

$$
f(x)=\frac{\varepsilon+(2 x+6)\left[\left(\frac{3}{2}\right)^{\mathrm{e}^{-2 x / \varepsilon}}-1\right]}{(x-3)^{2}}
$$

and boundary conditions

$$
y(0)=0 \quad \text { and } \quad y(1)=\log \frac{3}{2} \mathrm{e}^{-2 / \varepsilon} .
$$

Now, the function (6.9) is the exact solution of equation (6.8). We solve this equation with the three-point method $(k=3, m=1)$ and the four-point method $(k=4, m=2)$. The nonlinear system is solved by five iterations of a Newton-Raphson method. The results are presented in Figure 6.5. Again, the behavior of the error is the same as in Examples 6.1 and 6.3.

It is noteworthy that condition (1.3) is important for the convergence of the method. In a semilinear problem of the form $\varepsilon y^{\prime \prime}+b y^{\prime}+a(x, y)=f(x)$, the assumption $\frac{\partial a}{\partial y} \leq 0$ guarantees convergence. For example, the method did not converge for the problem $\varepsilon y^{\prime \prime}+2 y^{\prime}+\mathrm{e}^{y}=f(x)$.

7. Concluding remarks. In this paper we introduced a family of exponentially fitted difference schemes for singularly perturbed two-point boundary value problems. The schemes are derived from interpolating formulae for exponential sums. The consistency of the considered difference schemes follows from the error bounds given in [10]. Although this paper deals with exponential sums with a basis of the form $1, x, \ldots, x^{k-2}, \exp (\rho x)$, interpolating formulae may be applied to a wider class of exponential sums used in the literature, for example, sums with a basis $1, x, \ldots, x^{k-2}, \exp (\rho x), x \exp (\rho x), \ldots, x^{l} \exp (\rho x)$ or $1, x, \ldots, x^{k-2}, \exp (\rho x)$, $\exp (-\rho x)$. Consistency may follow from a generalization of the results in [10].

We illustrated this approach for three three-point difference schemes. All three methods are stable and convergent. As expected, the examples show that the central difference scheme gives the best approximation. The proposed methods are comparable to other exponentially fitted three-point methods such as, for example, the methods proposed in [1, 14]. Still, our methods have better properties than these two methods. 
For exponential fitting, we use the fitting parameter $\rho$ in (3.4) proposed for collocation methods by tension spline [9, 12]. All three methods are convergent for such a choice of $\rho$ as well as for the choice (3.5) commonly used in similar schemes. Moreover, the performed numerical tests indicate no difference between the approximations obtained by these two choices of $\rho$. Some methods from the literature use a uniform choice of $\rho$. In our case, a nonuniform choice guarantees convergence. Numerical tests show that a uniform choice of $\rho$ does not improve the method. Further, there are methods that use exponential fitting in the boundary layer only (for example [17]) or that behave well when exponential fitting is applied only in the boundary layer although the methods assume the exponential fitting over a whole interval (for example [9]). Such approach is in concordance with the fact that the solution of singularly perturbed problem exhibits strong exponential behavior only in the boundary layer. This is not possible in our case since exponential fitting on a whole interval is essential for the stability of the methods. Numerical tests show that the methods did not converge when exponential fitting was applied on the boundary layer alone.

We derived high-order schemes, higher than the seventh-order scheme given in [4]. The obtained results may be directly applied to schemes of higher order. We already have results for the stability of four-point schemes. However, a stability analysis of such schemes would be more complex.

Further, in the stability analysis we consider a singular perturbation problem with $b(x) \neq 0$ and $c(x)<0$. Numerical examples show that these schemes are applicable to equations where $b(x)=0$ or $c(x)=0$. In these cases, stability may be analyzed in the same way.

The proposed schemes are not $\varepsilon$-uniform convergent. However, convergence is guaranteed up to the point when the mesh width $h$ becomes smaller than $4(k-1) \varepsilon \ln (1 / \varepsilon) / b_{\min }$. If higher accuracy is required, then higher-order schemes may be used. The construction of a grid that would result in $\varepsilon$-uniform convergence is currently under investigation.

\section{REFERENCES}

[1] A. AWOKE AND Y. N. REDDY, An exponentially fitted special second-order finite difference method for solving singular perturbation problems, Appl. Math. Comput., 190 (2007), pp. 1767-1782.

[2] T. BosNer, Basis of splines associated with singularly perturbed advection-diffusion problems, Math. Commun., 15 (2010), pp. 1-12.

[3] D. BRAESS, Nonlinear Approximation Theory, Springer, Berlin, 1986.

[4] P. P. Chakravarthy, K. PhaneEndRa, AND Y. N. RedDy, A seventh order numerical method for singular perturbation problems, Appl. Math. Comput., 186 (2007), pp. 860-871.

[5] P. A. Farrell, A. F. Hegarty, J. J. H. Miller, E. O'Riordan, ANd G. I. Shishkin, Robust Computational Techniques for Boundary Layers, Chapman \& Hall, Boca Raton, 2000.

[6] J. E. FLAHERTY AND W. MATHON, Collocation with polynomial and tension splines for singularly-perturbed boundary value problems, SIAM J. Sci. Statist. Comput., 1 (1980), pp. 260-289.

[7] M. K. KaDAlBAJOO AND K. C. PATIDAR, A survey of numerical techniques for solving singularly perturbed ordinary differential equations, Appl. Math. Comput., 130 (2002), pp. 457-510.

[8] I. KAVČIČ, M. ROGINA, AND T. BOSNER, Singularly perturbed advection-diffusion-reaction problems: comparison of operator-fitted methods, Math. Comput. Simulation, 81 (2011), pp. 2215-2224.

[9] M. MARUŠIĆ, A fourth/second order accurate collocation method for singularly perturbed two-point boundary value problems using tension splines, Numer. Math., 88 (2001), pp. 135-158.

[10] - Limit properties of interpolation by exponential sums, in Proceedings of BAIL 2002, Perth, 2002, S. Wang and N. Fowkes, eds., University of Western Australia, Perth, 2002, pp. 183-188.

[11] - On ع-uniform convergence of exponentially fitted methods, Math. Commun., 19 (2014), pp. 545-559.

[12] M. MARUŠIĆ AND M. RoginA, A collocation method for singularly perturbed two-point boundary value problems with splines in tension, Adv. Comput. Math., 6 (1996), pp. 65-76.

[13] J. J. H. Miller, E. O'Riordan, AND G. I. Shishinin, Fitted Numerical Methods for Singular Perturbation Problems, World Scientific, River Edge, 1996.

[14] Y. N. RedDy AND P. P. Chakravarthy, An exponentially fitted finite difference method for singular perturbation problems, Appl. Math. Comput., 154 (2004), pp. 83-101. 
ETNA

Kent State University and

Johann Radon Institute (RICAM)

[15] H.-G. Roos, M. Stynes, And L. Tobiska, Numerical Methods for Singularly Perturbed Differential Equations, Springer, Berlin, 1996.

[16] G. I. SHISHKIN, Approximation of solutions of singularly perturbed boundary value problems with a parabolic boundary layer, U.S.S.R. Comput. Math. and Math. Phys., 29 (1989), pp. 1-10.

[17] J. Vigo-AgUiAR AND S. NATESAN, An efficient numerical method for singular perturbation problems, J. Comput. Appl. Math., 192 (2006), pp. 132-141. 